\title{
The expression of the PI3K/AKT/mTOR pathway in gastric cancer and its role in gastric cancer prognosis
}

\author{
This article was published in the following Dove Press journal: \\ OncoTargets and Therapy \\ I September 2015 \\ Number of times this article has been viewed
}

Objective: To analyze the correlation between sequential aspects of the phosphoinositide-3 kinase (PI3K)/AKT/mammalian target of rapamycin (mTOR) pathway by immunohistochemistry in the primary lesion of gastric cancer, clinicopathologic factors, and survival in Chinese patients to explore the role of sequential analysis of multiple targets in prognoses.

Methods: Immunohistochemistry was performed to examine the expression of PI3K, phosphorylated-AKT (p-AKT), and phosphorylated-mTOR (p-mTOR) in 59 primary lesion samples ranging from Stages I to IV after gastrectomy. The correlation between sequential expression of multiple targets, and clinicopathologic factors and survival was analyzed.

Results: The positive expression rates of PI3K, p-AKT, and p-mTOR were $49 \%, 58 \%$, and $56 \%$, respectively. There were eleven cases with three biomarkers positive (19\%), 22 cases with two biomarkers positive (37\%), and 19 cases with only one biomarker positive (32\%). Seven cases $(12 \%)$ were all negative. Multi-factorial Cox regression analysis showed that neural invasion, vascular invasion, size of the tumor, lymph nodes affected, metastasis, carbohydrate antigen 19-9 level, and PI3K/p-AKT/p-mTOR simultaneous expression were independent prognostic parameters. The risk of death for the cases with two biomarkers positive was 0.367 times that for the cases with three biomarkers positive $(P=0.166)$. The risk of death for the cases with only one biomarker positive was 0.105 times that for the cases with three biomarkers positive $(P=0.058)$. The risk of death for the cases with three biomarkers negative was 0.017 times that for the cases with three biomarkers positive $(P=0.022)$.

Conclusion: Our study generated the hypothesis that patients with gastric cancer with simultaneous expression of PI3K/p-AKT/p-mTOR had worse outcome. But we need more rigorous validation in a larger data set.

Keywords: gastric cancer, PI3K/AKT/mTOR, prognosis

\section{Introduction}

Over the past 10 years, great progress has been made in cancer research in terms of cancer-initiation mechanisms, new drug discovery, and therapeutic modes. ${ }^{1}$ We also found a number of signaling pathways that affect the development of gastric cancer, including human epidermal growth factor receptor 2 pathway, epidermal growth factor receptor pathway, vascular endothelial growth factor receptor pathway, HGF/c-Met pathway, and PD1/PD-L1 pathway. ${ }^{2}$ There are many drugs targeting these signaling pathways, some of which have showed survival benefit and have been used in clinical practice (trastuzumab, ramucirumab). The phosphoinositide-3 kinase (PI3K)-protein kinase (PKB/AKT)-mammalian target of rapamycin (mTOR) pathway is a canonical pathway involved in anti-apoptosis and prosurvival, which regulates a number of normal cellular activities, such as cell proliferation, survival, and migration. ${ }^{3}$ Previous 
studies examined the expression and phosphorylation of $\mathrm{PI} 3 \mathrm{~K}, \mathrm{AKT}$, and mTOR in gastric cancer tissues and discovered that these patients had abnormal expressions of this pathway. Patients testing positive for the abnormal expression of the PI3K/AKT/mTOR pathway were more likely to be in the late stages of gastric cancer with a lower survival rate. ${ }^{4-7}$ Several drugs targeting PI3K/AKT/mTOR pathways are currently in clinical trials for gastric cancer, including PI3K inhibitor (LY294002, MK2206), AKT inhibitor (MLN1117), and mTOR inhibitor (everolimus). ${ }^{8-11} \mathrm{PI} 3 \mathrm{~K}$ inhibitor and AKT inhibitor monotherapy showed promising effects in Phases I-II, while mTOR inhibitor failed to improve survival in the GRANITE-1 Stage III trial for advanced gastric cancer. It remains unclear and requires further investigation whether the negative results of GRANITE-1 were because of the limited function of the PI3K/AKT/mTOR pathway in gastric cancer progression, the lack of molecular markers for patient screening, or the limited efficacy of mTOR monotherapy. ${ }^{12,13}$ Recently, emerging evidence from clinical research has shown that combined inhibition of multiple pathways or multiple targets within the same pathway could further improve treatment efficacy. However, the sequential analysis of multiple targets within the PI3K/AKT/mTOR pathway and its correlation with clinicopathologic factors and prognosis were seldom reported either domestically or internationally.

EnVision (Dako Deutschland GmbH, Hamburg, Germany), an immunohistochemistry method, was applied to examine PI3K, phosphorylated-AKT (p-AKT), and phosphorylated-mTOR (p-mTOR) levels in 59 primary gastric cancer lesion samples after gastrectomy ranging from Stages I to IV (M0). The correlation between the sequential aspects of biomarker expression and clinicopathologic factors, as well as to survival, was analyzed.

\section{Materials and methods \\ Subjects of the study}

This study included 59 patients who received gastrectomy in Zhejiang Cancer Hospital, Hangzhou, People's Republic of China, from September 2004 to December 2004. All patients were screened according to the following criteria: 1) diagnosed as advanced gastric cancer by histopathology, 2) cancer tissue samples were collected, and 3) detailed clinical history and follow-up information were available. The study was approved by the medical ethics committee at Zhejiang Cancer Hospital. There were 44 males (75\%) and 15 females (25\%) in the study with a median age of 56 years (range: 28-75). The cases were categorized according to the depth of invasion
(T) (T1, eleven cases; T2, 14 cases; T3, 32 cases; and T4, two cases), the lymph node affected by the tumor (N) (N0, 13 cases; N1, 21 cases; N2, 19 cases; N3, six cases), and according to the gastric cancer TNM staging system (referring to the size of the original (primary) tumor and depth of invasion, lymph nodes affected, and metastasis) post-surgery (Stage I, 12 cases; Stage II, 13 cases; Stage III, 26 cases; and Stage IV [M0], eight cases). Gastric cancer TNM staging followed the standards set by the Union for International Cancer Control in 1997. The follow-up examinations were conducted from the surgery date until June 27, 2013. The median follow-up period was 107 (50-117) months. Both phone calls and letters were used in the follow-ups. Wrong and invalid phone numbers, failure to be contacted twice on different dates, and no reply to two follow-up letters were considered as a loss of connection. During the follow-up, 31 patients (53\%) died, three of whom died from unrelated causes.

\section{Reagents}

p-mTOR (Ser2448) (49F9) and PI3K P110a (C73F8) antibodies were purchased from Cell Signaling Technology (Danvers, MA, USA). p-AKT1/2/3 (Thr 308) antibody was purchased from Santa Cruz Biotechnology, Inc. (Heidelberg, Germany). All were rabbit polyclonal antibodies.

\section{Immunohistochemistry}

Immunohistochemical staining was done using the EnVision two-step immunohistochemistry kit (Dako Deutschland $\mathrm{GmbH}$ ). The samples were fixed in $4.0 \%$ formaldehyde solution and embedded in paraffin. The sample-containing paraffin was sliced into serial sections with a width of 5.0 $\mu \mathrm{m}$. The sections were then dewaxed and hydrated, followed by $3.0 \% \mathrm{H}_{2} \mathrm{O}_{2}$ treatment to block endogenous peroxidases, and recovered in microwave-heated citrate acid. The primary antibody was added and incubated overnight at $4.0^{\circ} \mathrm{C}$. The sections were then recovered at $37^{\circ} \mathrm{C}$ for 20 minutes, followed by secondary antibody incubation at room temperature for 30 minutes. The sections were developed using diaminobenzidine and nuclear staining with hematoxylin. Phosphate-buffered saline was added as the primary antibody in the negative control. No primary antibody or substitute was added into the blank control, and the known positive tissue section was used as the positive control.

\section{Result evaluation}

The results were blindly evaluated simultaneously by two pathologists. If there was disagreement, then the final conclusion was reached through discussion. PI3K-positive 
sections showed yellow or brownish staining distributed in the cytoplasm of tumor cells and/or epithelial cells around the tumor and, in some cases, in the nuclei; p-AKT-positive staining was yellow or brownish and was located in the nucleus or cytoplasm of the tumor cells; p-mTOR-positive staining was also yellow or brownish and was seen mostly in the cytoplasm of tumor cells and surrounding epithelial cells. Some p-mTOR-positive staining was also observed on the cell membrane and in intercellular substances. The results were graded on a scale from 0 to 4 by using a semi quantitative method according to the literature. Five fields (center, left, right, up, and down) of each section were selected under high magnification for cell counting, and the percentage of stain-positive cells in the same type of cells was recorded as the coloration rate. Grading according to the coloration rate was as follows: $0,0 \%$ stain-positive tumor cells; 1, stain-positive tumor cells $<10 \%$; 2 , stainpositive tumor cells $10 \%-50 \% ; 3$, stain-positive tumor cells $51 \%-80 \%$; and 4 , stain-positive tumor cells $>80 \%$ Grading according to stain intensity was as follows: 0 , no stain; 1 , light yellow; 2, brownish-yellow; and 3, brown. The score, ranging from 0 to 12 , was calculated as follows: score $=$ grade in stain intensity $\times$ grade in coloration rate. A score $\geq 4$ was considered to be positive. ${ }^{4,5,7}$

\section{Statistical analysis}

Statistical Package for the Social Sciences (SPSS) version 16.0 (SPSS Inc., Chicago, IL, USA) was used for the statistical analysis. The correlation between PI3K, p-AKT, and p-mTOR expression and patient clinicopathologic factors was analyzed using the $\chi^{2}$ test. The correlation between PI3K, p-AKT, and p-mTOR expression and survival was analyzed by using the Kaplan-Meier curve and log-rank test. Multifactorial analysis was conducted using Cox regression analysis. $P<0.05$ was considered as statistically significant.

\section{Results}

\section{Expression of PI3K, $\mathrm{p}-\mathrm{AKT}$, and $\mathrm{p}-\mathrm{mTOR}$ in gastric cancer tissues and the correlation with clinicopathologic factors}

The immunohistochemistry results showed that the positive rate of PI3K, p-AKT, and p-mTOR was 49\%, 58\%, and $56 \%$, respectively (Figures $1-3$ ). The correlation among the expression of PI3K, p-AKT, and p-mTOR and sex, age, anemia, differentiation, tumor diameter, alkaline phosphatase, C-reactive protein, lactate dehydrogenase

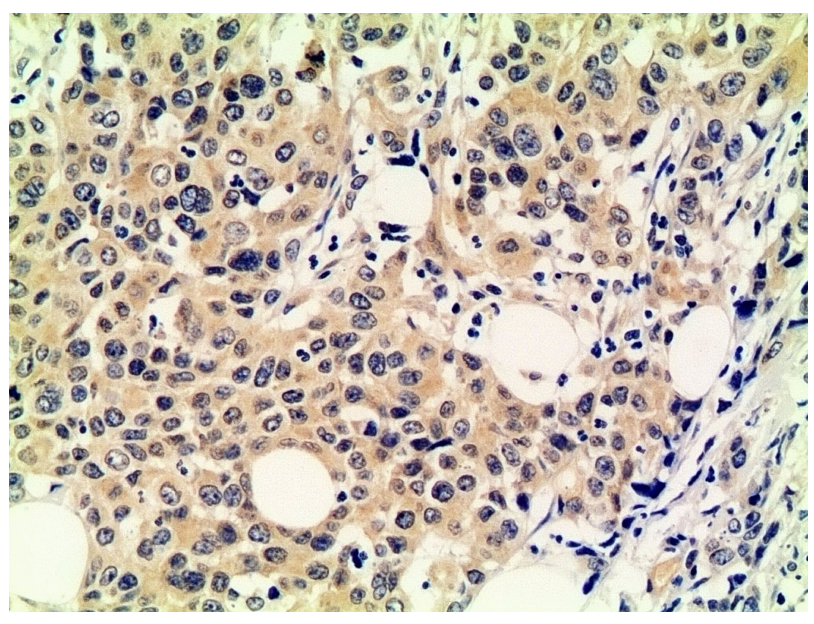

Figure I PI3K-positive expression in the primary lesion $(\times 200)$. Abbreviation: PI3K, phosphoinositide-3 kinase.

(LDH), carcinoembryonic antigen (CEA), carbohydrate antigen 19-9 (CA19-9), TNM staging, neural invasion, vascular invasion, and performance status grading was examined. LDH levels were significantly correlated with $\mathrm{PI} 3 \mathrm{~K} / \mathrm{p}-\mathrm{AKT} / \mathrm{p}-\mathrm{mTOR}$ expression. Six patients with increased LDH levels were positively expressed for all three biomarkers. Males had higher p-mTOR-positive rate than females (66\% vs $27 \%, P=0.008)$. The patients with a tumor diameter $<5.0 \mathrm{~cm}$ had higher PI3K-positive rate $(58 \%$ vs $32 \%, P=0.063)$ and $\mathrm{p}$-AKT-positive rate $(68 \%$ vs $37 \%, P=0.026)$. The patients with increased levels of CEA had higher PI3K-positive rate ( $77 \%$ vs $41 \%, P=0.023$ ) and lower p-mTOR-positive rate ( $31 \%$ vs $63 \%, P=0.038$ ). Sequential analysis of biomarkers showed no relationship with clinicopathologic factors.

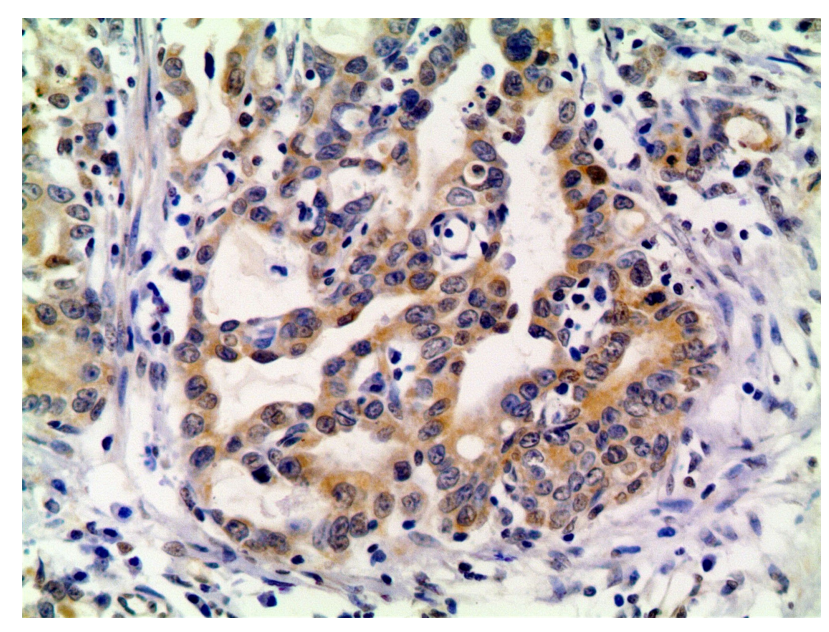

Figure 2 p-AKT-positive expression in the primary lesion $(\times 200)$. Abbreviation: $\mathrm{p}-\mathrm{AKT}$, phosphorylated-AKT. 


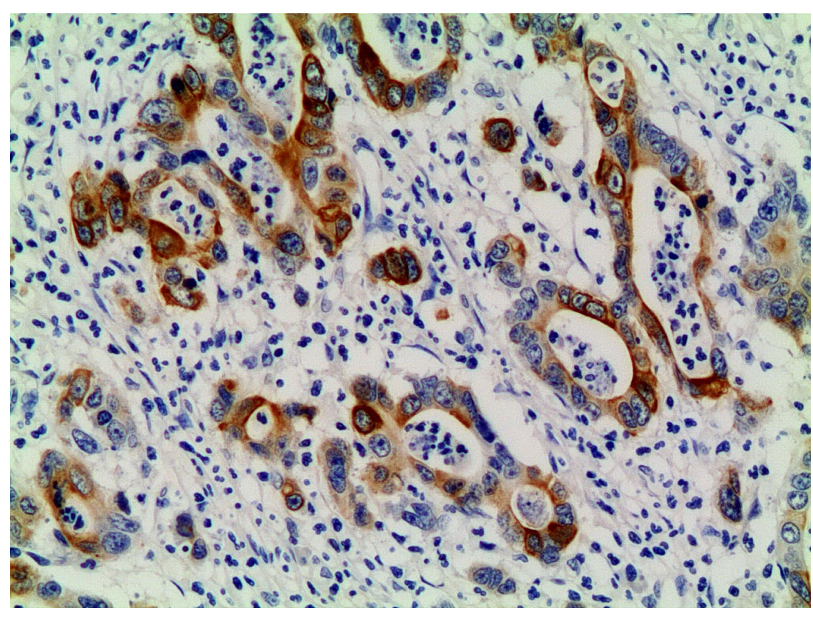

Figure 3 p-mTOR-positive expression in the primary lesion $(\times 200)$. Abbreviation: p-mTOR, phosphorylated mammalian target of rapamycin.

\section{Analysis of the correlation between clinicopathologic factors and survival}

Single-factorial analysis showed that performance status grading $>2$; neural and vascular invasion; deep tumor invasion; more lymph node metastasis; late TNM staging; and increased C-reactive protein, CEA, and CA19-9 levels were associated with poor survival. In single-factor analysis, neither individual biomarker nor sequential analysis of three biomarkers (expression of PI3K, p-AKT, and p-mTOR) was significantly correlated with survival (Table 1).

\section{Multi-factorial Cox regression}

Multi-factorial Cox regression showed that neural invasion, vascular invasion, TNM staging, CA19-9 level, and sequential analysis of $\mathrm{PI} 3 \mathrm{~K} / \mathrm{p}-\mathrm{AKT} / \mathrm{p}-\mathrm{mTOR}$ were independent prognostic factors. The risk of death increased by 2.773 when the tumor was ranked one stage higher according to TNM staging $(P=0.013)$; the risk of death for patients without neural invasion was 0.146 times that for patients with neural invasion $(P=0.001)$; the risk of death for patients without vascular invasion was 0.205 times that for patients with vascular invasion $(P=0.012)$; the risk of death for patients with normal CA19-9 was 0.212 times that for patients with high CA19-9 $(P=0.046)$. The risk of death for patients with two biomarkers positive, single biomarker positive, three biomarkers negative of PI3K/p-AKT/p-mTOR was 0.367 times $(P=0.166), 0.105$ times $(P=0.058), 0.017$ times $(P=0.022)$, respectively, that for patients with three biomarkers positive (Table 2).

\section{Discussion}

Gastric cancer is a heterogenous cancer, which may be classified into several distinct subtypes based on pathology and epidemiology, each with different initiating pathological processes and each possibly having different tumor biology. A classification of gastric cancer should be important to select patients who can benefit from the targeted therapies or to precisely predict prognosis. It has been reported that the abnormality in the PI3K/AKT/mTOR pathways is correlated with gastric cancer tumorigenesis and prognosis. According to The Cancer Genome Atlas study, activating PI3K mutations may be present in Epstein-Barr virus-associated gastric cancers and may be a potential driver in this subset. ${ }^{14,15}$ Our immunohistochemistry results showed that the percentages of positive PI3K, p-AKT, and p-mTOR were $49 \%, 58 \%$, and $56 \%$, respectively, which is consistent with previous reports. The correlation between the PI3K/p-AKT/p-mTOR pathway and LDH levels was found to be significant in our analysis of clinicopathologic factors. It was previously reported that there was a mutual promotion between a hypoxia-induced increase of LDH and the carcinogenic PI3K/AKT/mTOR pathway. In renal cancers, increases in serum LDH levels were a prognostic

Table I Difference in 3- and 5-year survival with various clinicopathologic factors

\begin{tabular}{|c|c|c|c|c|c|c|}
\hline Clinical factors & Cases (n) & Death $(n)$ & 3 years $(\%)$ & 5 years $(\%)$ & $x^{2}$ & $P$-value \\
\hline \multicolumn{7}{|l|}{ Sex } \\
\hline M & 44 & 23 & 54 & 52 & 0.019 & 0.890 \\
\hline $\mathrm{F}$ & 15 & 8 & 60 & 47 & & \\
\hline \multicolumn{7}{|l|}{ Age (years) } \\
\hline$<60$ & 33 & 15 & 63 & 57 & 2.046 & 0.153 \\
\hline$\geq 60$ & 26 & 16 & 46 & 42 & & \\
\hline \multicolumn{7}{|l|}{ PS grading } \\
\hline $0-1$ & 35 & 14 & 69 & 63 & 9.308 & 0.002 \\
\hline 2 & 24 & 17 & 38 & 32 & & \\
\hline \multicolumn{7}{|c|}{ Tumor diameter $(\mathrm{cm})$} \\
\hline$<5$ & 40 & 19 & 62 & 57 & 2. 197 & 0.138 \\
\hline$\geq 5$ & 19 & 12 & 42 & 37 & & \\
\hline
\end{tabular}


Table I (Continued)

\begin{tabular}{|c|c|c|c|c|c|c|}
\hline Clinical factors & Cases (n) & Death (n) & 3 years $(\%)$ & 5 years $(\%)$ & $\chi^{2}$ & $P$-value \\
\hline \multicolumn{7}{|c|}{ Differentiation status } \\
\hline High & 2 & I & 50 & 50 & 0.991 & 0.609 \\
\hline Medium & 26 & 13 & 65 & 57 & & \\
\hline Low & 31 & 17 & 48 & 45 & & \\
\hline \multicolumn{7}{|l|}{ Invasion depth } \\
\hline TI & II & 0 & 100 & 100 & 53.950 & 0.000 \\
\hline $\mathrm{T} 2$ & 14 & 5 & 85 & 77 & & \\
\hline T3 & 32 & 24 & 28 & 25 & & \\
\hline $\mathrm{T} 4$ & 2 & 2 & 0 & 0 & & \\
\hline \multicolumn{7}{|c|}{ Lymph node metastasis } \\
\hline No & 13 & 2 & 92 & 84 & 12.073 & 0.007 \\
\hline $\mathrm{NI}$ & 21 & 11 & 61 & 51 & & \\
\hline N2 & 19 & 14 & 32 & 25 & & \\
\hline N3 & 6 & 4 & 33 & 0 & & \\
\hline \multicolumn{7}{|l|}{ TNM staging } \\
\hline I & 12 & I & 100 & 92 & 22.713 & 0.000 \\
\hline II & 13 & 5 & 85 & 67 & & \\
\hline III & 26 & 19 & 31 & 26 & & \\
\hline IV & 8 & 6 & 25 & 0 & & \\
\hline \multicolumn{7}{|l|}{ Neural invasion } \\
\hline Yes & 17 & 14 & 24 & 18 & 12.206 & 0.000 \\
\hline No & 42 & 17 & 69 & 64 & & \\
\hline \multicolumn{7}{|l|}{ Vascular invasion } \\
\hline Yes & 20 & 16 & 30 & 23 & 13.618 & 0.000 \\
\hline No & 39 & 15 & 67 & 64 & & \\
\hline \multicolumn{7}{|l|}{ Anemia } \\
\hline No & 25 & 12 & 68 & 56 & 1.143 & 0.285 \\
\hline Yes & 34 & 19 & 47 & 47 & & \\
\hline \multicolumn{7}{|l|}{ CRP } \\
\hline Normal & 46 & 21 & 65 & 58 & 7.817 & 0.005 \\
\hline High & 13 & 10 & 23 & 23 & & \\
\hline \multicolumn{7}{|l|}{ AKP } \\
\hline Normal & 56 & 29 & 57 & 51 & 0.268 & 0.605 \\
\hline High & 3 & 2 & 33 & 33 & & \\
\hline \multicolumn{7}{|l|}{ LDH } \\
\hline Normal & 53 & 29 & 55 & 48 & 0.354 & 0.552 \\
\hline High & 6 & 2 & 67 & 67 & & \\
\hline \multicolumn{7}{|l|}{ CEA } \\
\hline Normal & 46 & 20 & 65 & 61 & 8.206 & 0.004 \\
\hline High & 13 & II & 19 & 10 & & \\
\hline \multicolumn{7}{|l|}{ CA199 } \\
\hline Normal & 49 & 23 & 63 & 57 & 4.573 & 0.032 \\
\hline High & 10 & 8 & 20 & 20 & & \\
\hline \multicolumn{7}{|l|}{ PI3K } \\
\hline Negative & 30 & 14 & 67 & 56 & 0.885 & 0.347 \\
\hline Positive & 29 & 17 & 45 & 45 & & \\
\hline \multicolumn{7}{|l|}{$\mathrm{p}-\mathrm{AKT}$} \\
\hline Negative & 25 & 14 & 59 & 52 & 0.186 & 0.666 \\
\hline Positive & 34 & 17 & 48 & 48 & & \\
\hline \multicolumn{7}{|l|}{ p-mTOR } \\
\hline Negative & 26 & 15 & 53 & 45 & 0.255 & 0.613 \\
\hline Positive & 33 & 16 & 58 & 54 & & \\
\hline \multicolumn{7}{|c|}{ PI3K, p-AKT, mTOR } \\
\hline Three positive & 11 & 4 & 64 & 64 & 2.286 & 0.515 \\
\hline Two positive & 22 & 14 & 45 & 39 & & \\
\hline Single positive & 19 & 10 & 58 & 53 & & \\
\hline Three negative & 7 & 3 & 57 & 37 & & \\
\hline
\end{tabular}

Abbreviations: AKP, alkaline phosphatase; CAI99, carbohydrate antigen 19-9; CEA, carcinoembryonic antigen; CRP, C-reactive protein; LDH, lactate dehydrogenase; F, female; M, male; PI3K, phosphoinositide-3 kinase; p-AKT, phosphorylated-AKT; p-mTOR, phosphorylated mammalian target of rapamycin; PS, performance status. 
Table 2 Multi-factorial Cox regression of the correlation between clinicopathologic factors and survival

\begin{tabular}{llllll}
\hline Clinical factors & B & SE & Wald & P-value & RR (95\% CI) \\
\hline Neural invasion & -1.924 & 0.577 & 11.126 & 0.001 & $0.146(0.047-0.452)$ \\
Vascular invasion & -1.586 & 0.629 & 6.354 & 0.012 & $0.205(0.060-0.703)$ \\
TNM staging & 1.020 & 0.409 & 6.206 & 0.013 & $2.773(1.243-6.187)$ \\
CEA & $1.55 I$ & 0.812 & 3.649 & 0.056 & $4.718(0.960-23.171)$ \\
CAI99 & -1.552 & 0.777 & 3.986 & 0.046 & $0.212(0.046-0.972)$ \\
PI3K/P-AKT/P-mTOR & & & 5.231 & 0.156 & \\
PI3K/p-AKT/p-mTOR any two positive* & -1.003 & 0.725 & 1.916 & 0.166 & $0.367(0.089-1.518)$ \\
PI3K/P-AKT/P-mTOR single positive* & -2.259 & 1.191 & 3.596 & 0.058 & $0.105(0.010-1.079)$ \\
PI3K/P-AKT/P-mTOR three negative* & -4.060 & 1.778 & 5.215 & 0.022 & $0.017(0.001-0.562)$ \\
\hline
\end{tabular}

Note: *Compared with PI3K/p-AKT/p-mTOR three markers positive.

Abbreviations: CA 199, carbohydrate antigen 19-9; CEA, carcinoembryonic antigen; PI3K, phosphoinositide-3 kinase; p-AKT, phosphorylated-AKT; p-mTOR, phosphorylated mammalian target of rapamycin; $\mathrm{Cl}$, confidence interval; SE, standard error; RR, relative risk.

factor for survival as well as a predictor for the efficacy of temsirolimus, an mTOR/TORC1 inhibitor. ${ }^{16}$ In our study, all three indices were $100 \%$ expressed in patients with increased LDH levels, which indicate that, in gastric cancer, there is a close association between LDH elevation and the PI3K/ AKT/mTOR pathway. Whether LDH levels could serve as a predictor for the efficacy of PI3K/AKT/mTOR pathway inhibitors requires further investigation. The risk of death for patients with two biomarkers positive, single biomarker positive, three biomarkers negative of $\mathrm{PI} 3 \mathrm{~K} / \mathrm{p}-\mathrm{AKT} / \mathrm{p}-\mathrm{mTOR}$ was 0.367 times $(P=0.166), 0.105$ times $(P=0.058), 0.017$ times $(P=0.022)$, respectively, that for patients with three biomarkers positive. These result raised the hypotheses that sequential analysis may have stronger prognostic significance, it is worthy of further research to verify the direction. Basic research shows that the mTOR inhibitor could activate PI3K/AKT and MAPK pathways through negative feedback, attenuating its anticancer effect. ${ }^{17,18}$ Hence, single-targeted inhibitors tend to have limited efficacy and introduce tolerance. Joint inhibition of multiple pathways or multiple targets in the same pathway has gained more and more attention in recent years. VS-5584 is a potent mTOR and PI3K inhibitor with low molecular weight. It has shown great antitumor growth effects in many cancer cell types, including gastric cancer, and in experiments on mice. ${ }^{19}$ If our hypothesis is verified in a larger sample of the population, then it can be used to predict patients with different prognosis and screen patients who may benefit from joint inhibition of multiple targets in the future.

There were limitations in our study because it was retrospective using a small sample size. What to do next? First, the conclusion of our research needs to be further evaluated in future larger studies. Second, we examined only the protein expression levels of the PI3K/AKT/mTOR pathway without testing gene amplification and mutation, which did not fully reflect any gene alterations in the pathway; this also requires further investigation.

\section{Acknowledgment}

This work was supported by the Natural Scientific Foundation of Zhejiang Province, People's Republic of China (LY14H160008).

\section{Author contributions}

Hongming Pan was responsible for substantial contributions to conception and design. Qi Xu was responsible for acquisition of data, or analysis and interpretation of data. Jieer Ying was responsible for drafting the article or revising it critically for important intellectual content. Bixia Liu and Lei Chen were responsible for all aspects of the work in ensuring that questions related to the accuracy or integrity of any part of the work are appropriately investigated and resolved. Gu Zhang was responsible for immunohistochemical detection. All authors contributed toward data analysis, drafting and revising the paper and agree to be accountable for all aspects of the work.

\section{Disclosure}

The authors report no conflicts of interest in this work.

\section{References}

1. Sjoquist KM, Zalcberg JR. Gastric cancer: past progress and present challenges. Gastric Cancer. 2015;18(2):205-209.

2. Aprile G, Giampieri R, Bonotto M, et al. The challenge of targeted therapies for gastric cancer patients: the beginning of a long journey. Expert Opin Investig Drugs. 2014;23(7):925-942.

3. Vivanco I, Sawyers CL. The phosphatidylinositol 3-Kinase AKT pathway in human cancer. Nat Rev Cancer. 2002;2(7):489-501.

4. Tian WY, Chen WC, Li R, Liu L. Markers CD40, VEGF, AKT, PI3K, and S100 correlate with tumor stage in gastric cancer. Onkologie. 2013; 36(1-2):26-31.

5. Sukawa Y, Yamamoto H, Nosho K, et al. Alterations in the human epidermal growth factor receptor 2-phosphatidylinositol 3-kinase-v-Akt pathway in gastric cancer. World J Gastroenterol. 2012;18(45):6577-6586. 
6. Li M, Sun H, Song L, Gao X, Chang W, Qin X. Immunohistochemical expression of mTOR negatively correlates with PTEN expression in gastric carcinoma. Oncol Lett. 2012;4(6):1213-1218.

7. Yu G, Wang J, Chen Y, et al. Overexpression of phosphorylated mammalian target of rapamycin predicts lymph node metastasis and prognosis of Chinese patients with gastric cancer. Clin Cancer Res. 2009; 15(5):1821-1829.

8. Xing CG, Zhu BS, Fan XQ, et al. Effects of LY294002 on the invasiveness of human gastric cancer in vivo in nude mice. World J Gastroenterol. 2009;15(40):5044-5052.

9. Yap TA, Yan L, Patnaik A, et al. First-in-man clinical trial of the oral pan-AKT inhibitor MK-2206 in patients with advanced solid tumors. J Clin Oncol. 2011;29(35):4688-4695.

10. Hirai H, Sootome H, Nakatsuru Y, et al. MK-2206, an allosteric Akt inhibitor, enhances antitumor efficacy by standard chemotherapeutic agents or molecular targeted drugs in vitro and in vivo. Mol Cancer Ther. 2010;9(7):1956-1967.

11. Li B, Wang L, Chi B. Upregulation of periostin prevents P53-mediated apoptosis in SGC-7901 gastric cancer cells. Mol Biol Rep. 2013; 40(2):1677-1683.

12. Ohtsu A, Ajani JA, Bai YX, et al. Everolimus for previously treated advanced gastric cancer: results of the randomized, double-blind, phase III GRANITE-1 study. J Clin Oncol. 2013;31(31):3935-3943.
13. Meric-Bernstam F, Akcakanat A, Chen H, et al. PIK3CA/PTEN mutations and Akt activation as markers of sensitivity to allosteric mTOR inhibitors. Clin Cancer Res. 2012;18(6):1777-1789.

14. Chen J. Roles of the PI3K/Akt pathway in Epstein-Barr virus-induced cancers and therapeutic implications. World J Virol. 2012;1(6): 154-161.

15. Cancer Genome Atlas Research Network. Comprehensive molecular characterization of gastric adenocarcinoma. Nature. 2014; 513(7517):202-209.

16. Armstrong AJ, George DJ, Halabi S. Serum lactate dehydrogenase predicts for overall survival benefit in patients with metastatic renal cell carcinoma treated with inhibition of mammalian target of rapamycin. $J$ Clin Oncol. 2012;30(27):3402-3407.

17. Albert L, Karsy M, Murali R, Jhanwar-Uniyal M. Inhibition of mTOR activates the MAPK pathway in glioblastoma multiforme. Cancer Genomics Proteomics. 2009;6(5):255-261.

18. Carracedo A, Ma L, Teruya-Feldstein J, et al. Inhibition of mTORC1 leads to MAPK pathway activation through a PI3K-dependent feedback loop in human cancer. J Clin Invest. 2008;118(9):3065-3074.

19. Hart S, Novotny-Diermayr V, Goh KC, et al. VS-5584, a novel and highly selective PI3K/mTOR kinase inhibitor for the treatment of cancer. Mol Cancer Ther. 2013;12(2):151-161.
OncoTargets and Therapy

\section{Publish your work in this journal}

OncoTargets and Therapy is an international, peer-reviewed, open access journal focusing on the pathological basis of all cancers, potential targets for therapy and treatment protocols employed to improve the management of cancer patients. The journal also focuses on the impact of management programs and new therapeutic agents and protocols on

\section{Dovepress}

patient perspectives such as quality of life, adherence and satisfaction. The manuscript management system is completely online and includes a very quick and fair peer-review system, which is all easy to use. Visit http://www.dovepress.com/testimonials.php to read real quotes from published authors. 